\title{
Ontology of the wave function and the many-worlds interpretation
}

\section{Introduction}

Quantum theory is about a century old, but as the existence of this volume shows, we are far from consensus about its interpretation. Science does not develop in a straight line. For a decade, the quantum theory had no real basis, only phenomenological equations found by Bohr, who made many of us believe until today that quantum mechanics cannot be understood. The relativistic generalizations of Schrodinger equation, however, provide a complete elegant physical theory fully consistent with experimental data with precision of up to 10 significant digits. The situation today is much better than at the time of Lord Kelvin's speech at 1900 in which he was arguing that physics is almost finished accept for solving "two clouds" which were later to become the theory of relativity and quantum theory. This, and the fact that quantum equations describe several outcomes for quantum measurements, although we always see just one, are probably the main reasons why contemporary physicists are reluctant to state that physics is close to be finished.

To deal with this second problem we either have to add something to the wave equation, but no proposal attractive enough to become a consensus was found, or to admit that what we see is only a tiny part of what is and that there are multiple parallel worlds similar to ours. I find that this last option is the only reasonable one and I hope it will reach the consensus in a foreseeable future.

\section{A toy model of classical mechanics}

Before discussing quantum mechanics, which is clearly not simple since we are very far from consensus about its interpretation, I would like to discuss a toy model of classical mechanics. It is an implementation of Laplace Universe:

We ought to regard the present state of the universe as the effect of its antecedent state and as the cause of the state that is to follow. An intelligence knowing all the forces acting in nature at a given instant, as well as the momentary positions of all things in the universe, would be able to comprehend in one single formula the motions of the largest bodies as well as the lightest atoms in the world, provided that its intellect were sufficiently powerful to subject all data to analysis; to it nothing would be uncertain, the future as well as the past would be present to its eyes. The perfection that the human mind has been able to give to astronomy affords but a feeble outline of such an intelligence.

[Laplace 1820]

I assume that the clouds Lord Kelvin talked about do not exist. Newton laws and Maxwell equations are somewhat different and they provide a consistent theory for microscopic particles 
moving on continuous trajectories, which, using the methods of statistical mechanics, explains well all experimental data. It is also an assumption about the experimental data, since we know that actually observed data cannot be explained by a classical model.

In the model we consider the three-dimensional space is given. There are particles moving in well defined trajectories and fields spread out in space. Particles create fields which propagate in space and change motion of other particles present in locations with nonvanishing fields. The laws of creation and propagation of fields explain the existence of stable rigid objects and everything else (including ourselves) which we experience with our senses. The behavior of objects is deterministic, free will is an illusion.

Like in actual physics, our model can be presented in a different way. There is a point in configuration space of all particles and configuration of fields fulfilling some global equation of extremal action. The global laws provide the same solution for trajectories. Both explanations are acceptable, but I feel that it is the first presentation with fields locally acting on particles moving on trajectories in 3D space that is a more convincing explanation of the world. Existence of a global mathematical representation is important, but it hides the local causal story which is what is considered as an explanation of motion of micro systems as well as our behavior.

It seems to me that in a counterfactual universe with successful classical physics as described above there will be no philosophical controversy how to describe reality. Particle trajectories governed by local forces through fields in three dimension would be a clear consensus.

\section{Ontology of collapsed wave function}

Our world is not classical. Numerous experiments, e.g. particle interference, contradict this picture. Moreover, Bell-type correlations show that no classical-type local theory, i.e. a theory which locally predicts a single outcome for each possible experiment can reproduce observed correlations. We have a new theory, quantum mechanics. It is true that the community of physicists working in the field of foundations of quantum mechanics have no consensus of its interpretation. Many of them (like me) are certain that their favorite view is a satisfactory (or even an excellent) solution, but each separate group is a small minority, so the message of the community as a whole is that currently there is no good solution. There are many, also outside the foundations community, who feel that we need the correct interpretation, but that it is different from all current proposals. However, the majority of physicists really think that the problem does not exist and that textbook quantum mechanics is satisfactory. It tells us that every time we perform a quantum measurement there is a collapse of the quantum wave function and that the collapsed wave function describes well all what we see around. Von Neumann proved that the tough question, when exactly collapse occurs, need not be answered since whenever we put the cut between classical and quantum, we will observe no contradiction with our experience. A vague statement according to which all "macroscopic" objects are "well" localized provides a satisfactory criterion. 
Von Neumann collapse is ad hoc without any concrete mechanism. In physical collapse theories, Pearle (1976), Ghirardi et al. (1986), Diosi (1987), Penrose (1996), the collapsed wave function is not completely identical to that of von Neumann, but it is very close, so also proponents of physical collapse theories consider the collapsed wave function as a satisfactory description of what we see. Apparently it is the promotion of the wave function ontology in the configuration space by Albert (2013) which led to strong criticism. Maudlin (2013) understandably complained: how a mathematical object in high dimensional space can represent our experience in three dimensions?

The key to answering this question is the understanding that our experience supervenes on macroscopic objects. We do not directly experience the electron wave function in the atoms of our body. Parts of our body and our neurons are macroscopic bodies. We needed configuration space because of entanglement. While for describing classical particles we had a choice between one point in $3 \mathrm{~N}$ dimensional space or $\mathrm{N}$ points in three dimensional space, in the quantum case with entanglement the second option does not exist, entanglement requires multidimensional space: complete descriptions of all particles separately do not provide complete description of entangled particles. But entanglement of quantum systems does not exist for macroscopic systems. In von Neumann approach it is absent by definition and in physical collapse theories, the mechanism removes entanglement of macroscopic objects very quickly. Without entanglement, we can describe all objects in three dimensions. To summarize, analysis of the process of obtaining experience from our senses when the universe is described by a collapsed wave function of a textbook or by the collapsed wave function of a physical collapse proposal provides an explanation similar to that of classical physics: (macroscopic) objects moving in three-dimensional space locally trigger sensory organs of observers living in this three dimensional space.

Current (quantum) physical theory based on minimal action does not always provide a simple local explanation of behavior of particles as our gedanken classical model. The basic concept of quantum theory is potentials which are not measurable locally. Classical physics also uses potentials, but they are just auxiliary tools helping to solve various problems more efficiently. In classical physics operational meaning of potentials is that their derivatives provide observable forces. In quantum mechanics Aharonov-Bohm effect (1959) teaches us that potentials provide a direct physical effect: particle moving in a free-field region behaves differently depending on the potentials present there. At least for myself, I resolved the difficulty, Vaidman (2012a), finding a local explanation of the $\mathrm{AB}$ effect based on considering the source of the potential to be quantum and taking into account entanglement between the electron and the source. This explanation removed the proof that quantum theory based on direct action of local fields cannot exist. I opened the way to such a theory, which, if constructed, will be conceptually more satisfactory than the present one.

\section{Against collapse}


Nonlocality of the Aharonov-Bohm effect makes a physical explanation more sophisticated, but the main ugly scar of quantum mechanics is collapse. I want to believe that today's physics by and large correctly explains everything we see around. For this belief I need two ingredients. First, that predictions of the theory correspond to what we see, i.e. to be confirmed by experimental results. Second, that the theory is elegant enough to believe that this is the description of Nature. We have had incredible success of the first part. In all cases we can calculate and measure, there is a complete agreement and sometimes, with astronomical precision of more than ten digits. There are no "clouds" similar to those seen by Lord Kelvin in classical physics. There is also incredible success of the theory. All basic laws of physics can be written on a t-shirt. Today's quantum mechanics (even with potentials) is a good theory: it is deterministic and it does not have action at a distance. Only the collapse spoils it. It is the only random process in physics.

The collapse is sometimes considered to be in "peaceful coexistence" with special relativity. Indeed, we cannot send superluminal signals using the collapse process. But it is an action at a distance. A particle described by a superposition of wave packets in two separate locations leads to action at a distance when the presence of a particle in one location is measured. The complete quantum description of a region of space with the second wave packet before the measurement is a mixed state. It has the following operational meaning: everyone, everywhere, have a welldefined betting strategy on the result of a measurement testing the presence of the particle in this place. Measurement in the location of the first wave packet changes the situation immediately. Obviously so, if the wave function is an ontic entity, since it is changed by a remote action, but also if it is only an epistemic concept. There is a change in operational meaning. If I and you are in the location of the first wave packet, you are ready to bet with me on the result of the remote measurement, but this changes immediately after you observe that I made a measurement on the first wave packet.

Contrary to widespread belief, there is no evidence for collapse of the wave function. It is true that for every quantum experiment we observe one outcome, while a theory without collapse has a wave function corresponding to several outcomes. But to be considered evidence, we need for the theory to predict a different experience in case the collapse takes place or not. The theory does not predict this. In fact, looking on a wave function of quantum mechanics without collapse it is not easy to make any prediction about experience. We need to add some postulates to connect the ontology with our experience.

\section{Connecting ontology with our experience}

Let us start the analysis with an interpretation of quantum mechanics where the connection is simple. In Bohmian mechanics, Goldstein (2017), the postulate is that experience supervene on Bohmian positions of particles and the wave function is only a pilot wave of these particles. 
Then, the Bohmian picture of positions of all particles is similar to the gedanken classical theory I sketched above. All particles provide a familiar picture of what we see around us drawn in three dimensions in a pointillist style. Bohmian particles, according to Bohmian mechanics postulates, are distributed in the locations of a nonvanishing wave function according to the Born rule. But the size of the atoms and thus the size of the wave packets of elementary particles are so small that the difference between locations of Bohmian points, and centers of particle's wave packets is inessential. Thus, considering the centers of the wave functions of particles in the collapsed wave function also provides a familiar picture. The fact that most of elementary particles are entangled and do not have a pure state, does not spoil this picture. Their mixed states are still well localized on the atom scale, so the expectation value of their positions are not far from corresponding to Bohmian positions.

Bohmian particles describe a world which looks like the one we observe and also the collapsed wave function does. Because the wave functions of macroscopic objects are well localized, the picture drawn by the expectation values of the position vectors of all particles of these objects also provides a familiar picture. The particles are very close to each other, so we do not observe the points. We observe the smoothed picture of everyday objects: tables, cats, people, etc.; the collapsed wave function provides a familiar picture of the world. The theory has a tacit assumption, a postulate: our everyday experience supervenes on the collapsed wave function. Since the picture drawn by the particles and the picture we draw based on our experience are so similar, we usually forget that we make an assumption connecting the formalism with experience. The theory is supposed to describe our experience. In this theory there is only one picture which looks like the world we see, we assume that there is only one world, so naturally we connect them. But the postulate is needed, since there can be other options: we can imagine presence of Bohmian particles also in a theory which makes collapses and attach experience to Bohmian particles. It is a different rule, although it seems to provide essentially the same experience.

\section{Connecting ontology with experience in the framework of the MWI}

In quantum mechanics without collapse we must add a postulate to connect to our experience since mathematics does not provide a (unique) picture corresponding to what we see around.

My postulate will be the following. The universe with non-collapsing wave function corresponds to multiple experiences. Each experience should correspond to at least one world, the definition of the concept of a world does not allow multiple simultaneous experiences of a person in a world. To connect the wave function of the Universe to our experience we need first to decompose the wave function of the Universe to a superposition of wave functions of worlds.

$$
|\Psi\rangle_{\text {UNIVRSE }}=\sum_{i} \alpha_{i}\left|\psi_{i}\right\rangle_{\text {WORLD }}
$$


The wave function of a world $\left|\psi_{i}\right\rangle$ must be of a particular type. Probably the most informative definition is that this is the type of wave functions which might appear in the textbook: "collapsed wave function of the Universe". Without relying on textbook definition, this type of wave function can be defined by the property that all macroscopic objects must be well localized. Both definitions are vague. We usually require rigor and precision in our theories. However, it must be so when we consider exact sciences. The many-worlds interpretation (MWI) has two clearly separated parts: i) a precise physical theory of evolution of the wave function of the Universe $|\Psi\rangle_{\text {UNIVERSE }}$ and ii) connection of this universal wave function to our experience(s). In classical theory, in Bohmian theory, in quantum mechanics with collapse, the separation between the two parts of theory was not emphasized because the second part was very simple: the connection is natural and obvious in contrast to the MWI, where the second part is significant. But I believe that the connection implicitly taken in single-world theories can be directly adopted to the MWI, and vagueness of the splitting of the worlds is much less problematic than the vagueness of the answer to the question when does collapse occur. Our experiences cannot be described in terms of exact science. So, it is understandable and acceptable that the concepts belonging to the second part of the theory are not rigorously defined. The wave function of a world has the following form

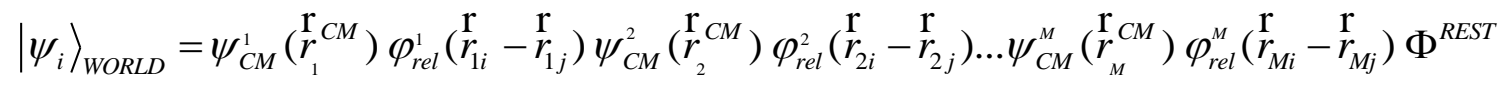

It is a product state of wave functions $\psi_{C M}^{k}\left({ }_{k}^{r}{ }^{C M}\right)$ of centers of mass of macroscopic objects times entangled states of relative coordinates of these objects $\varphi_{r e l}^{k}\left(r_{k i}-r_{k j}\right)$ times the wave function $\Phi^{R E S T}$ of the remaining particles which are not part of macroscopic objects. The terms "macroscopic", "well localized" might be chosen as more or less "fine grained", so the decomposition (1) is only approximately defined.

It seems to me that I can stop here. Textbook quantum theory is a well established and well tested theory which explains well everything we see around. It includes the unphysical process of collapse which makes it very difficult to believe that it is true. I remove the collapse and use the same postulate of connection to our experience. Now, the theory is a good physical theory (deterministic, no action at a distance and no ad hoc rules). The postulate of the correspondence of the experience with the wave function of the type of the universal wave function of a collapsed world makes the experience by fiat identical to that of an experience of an observer living in a Universe with collapsing wave functions. However, I know that this picture is (still) not in a consensus. I need to persuade the community that it is consistent.

\section{Naïve criticism}

The most naïve criticism is that we do not have experimental evidence for the MWI. How all these numerous worlds can be present in the same space as our world without usu noticing their presence? Equations tell us that there is no interaction between objects in different worlds. 
Moreover, we see in the laboratory that for micro systems, there is no scattering between wave packets of the same electron. Note that we also have no experimental evidence for the existence of collapse. Testing MWI versus collapse theories will be the performance of a quantum experiment up to a stage that the collapse proponents accept that collapse must have happened and then to undo the experiment Deutsch (1986) Vaidman (1998). If we get the original state every time we perform this procedure, it is proof that collapse did not take place. Until today there is no sign of a collapse, but we are very, very far from a decisive experiment. If collapse proponents will claim that collapse happens only after we write the result of a quantum experiment in a notebook, it can never be tested. Of course, it is the MWI that cannot be proved. If collapse exists on an early stage, it will be observed (Although it will not be easy to persuade opponents that the explanation of the signal is not a failure of experimentalists to prevent decoherence).

Slightly less naive criticism is the problem of preferred basis. Mathematically, one can decompose the wave function of the Universe into a superposition of orthogonal components not just as (1), but in many other ways which will not provide a familiar world's picture in every branch. So, the critics might say that the proposal is circular: I define by fiat what I want to explain. First, a simple definition which is confirmed by observation sounds to me a legitimate strategy. But there is also a more specific answer. The basis of the decomposition is indeed preferred. Until now I have not mentioned time evolution. Everything was considered at a particular moment. But we cannot experience anything at zero time. We need an order of 0.1 seconds to identify our experience. Thus, the world needs some finite time to be defined. The world has to be stable at least on the scale of seconds. Locality of interactions in nature ensures that only the decomposition to wave functions corresponding to well localized macroscopic objects can be stable. A quantum state describing the superposition of a macroscopic object in separate locations with a particular phase evolves almost immediately into a mixture which has a large component with a different phase. This obvious fact is analyzed in numerous papers with a buzz word "decoherence".

\section{The probability problem}

The most difficult issue is probability. The idea of parallel worlds is relatively new for humanity, so language and philosophy describing this situation is not well developed. Lewis (1986) provided some insight, but the philosophical issues related to plurality of worlds are far from clear.

The MWI I advocate has no uncertainty, randomness, objective chance which makes the concept of probability difficult. In the collapse theory there is a clear meaning of probability: the event happened while it could also not have happened, in the MWI such meaning does not exist. The event happened in this world, but there is no alternative, it could not be otherwise. I want to say that the traditional concepts of probability are not applicable in the framework of the MWI when we consider the outcomes of quantum experiments to be performed. Every time we perform a 
quantum experiment and it seems to us that a single result is obtained, all possible outcomes are obtained, each in a different world. There is no meaning to the question: in which world I will end up? In some sense I will be in all. In no sense, I will be in a particular one.

Still, we have to explain our experience of apparent random behavior and the frequency pattern of the results of quantum experiments. My claim remains that there is no difference between my experience if I live in one of the MWI worlds and my experience if I live in the only world of the Universe with collapses on every measurement. How to reconcile the difference between the existence of a probability concept and identity of experience? To avoid the difficulty there are proposals to introduce uncertainty in the MWI and provide the meaning for probability that I will end up in a world with a particular outcome. In my view, adding uncertainty to the theory spoils it. The wave function of the Universe is supposed to be the whole physical ontology and it does not have any pointer moving from one world to another.

But do we really have a problem here? The fact that there is no meaning for probability of the result of a future measurement does not contradict the claim of identical experiences. The standard approach to probability is to consider events which will happen, but testing probability claims relies on records of experiments in the past. Frequencies of the outcomes of repeated identical measurements performed in the past. So even in the framework of collapse theory, probability assignments are confirmed or refuted by our experiences in the past. Thus, the difficulty of the MWI to introduce the concept of probability for future outcomes is not relevant.

Assignment of probability for future experiment relies on an additional assumption even in the framework of a collapse theory. We assume that nature will not change its laws. Another way to make predictions about future measurement is to assume, that after performing the measurement, the frequency of the measurement results fits our probability assignment. This approach may also be applied in the framework of the MWI. We expect that within the world the frequencies of the results of past measurements will correspond to the probability assignment.

\section{Probability of self-location}

The counterpart of the Born Rule in the framework of the MWI is sometimes named the BornVaidman Rule:

The probability of self-location of an observer in a particular world is proportional to the measure of existence of this world.

The (somewhat controversial) term of "measure of existence", Vaidman (1998), Groisman et al. (2013), is just the square of the amplitude of the coefficient of corresponding wave function in decomposition of the Universal wave function (1). This rule explains correspondence of experimental data of quantum experiments with statistics predicted by the Born Rule. 
Probability is a very controversial philosophical concept. Frequency of particular outcomes in experimental records is just one approach. Another approach is readiness to put bets on the results of experiments according to their probability to happen. Since in the MWI all possible outcomes happen, there is difficulty to understand this aspect of probability. In 1998 I proposed a gedanken experiment which allows sensible betting also in the framework of the MWI, tightening the connection of the Born-Vaidman rule with the concept of probability. In my proposal, after arranging quantum experiment the observer takes a sleeping pill, Vaidman (1998). During observer's sleep the experiment is performed and the observer is moved to various rooms with identical interior according to the results of the experiment. When he is awakened, he understands that there are several copies of him in different rooms corresponding to the results of the experiment, however, he does not know who he is and thus he does not know the result of the experiment in his world. He might be given the wave function of the Universe; he still has ignorance which allows him to bet. Awakened decedents of the experimentalist preparing the experiment have a genuine ignorance concept of probability, similar to that of an experimentalist in the collapsing universe. Only the questions are different: the latter asks: What will be the result of the experiment? The former asks: In which world (defined by the result of the experiment) am I?

Note that all descendants have the same information and thus provide identical assignments for probabilities of different outcomes. This allows to define a rational betting strategy for an experimentalist before the experiment. Instead of probability postulate he has the caring principle, Vaidman (1998), Greaves (2004):

The experimentalist cares about his descendants in proportion to their measure of existence.

The justification of this principle for our betting example is the following. Every descendant will have a genuine probability concept and would like to have a bet according to a particular probability assignment. Since the descendants will get the reward of the bet, the experimentalist, naturally caring for his descendants, has a rational reason to put the bet for the results of the experiment. Tappenden (2011) suggested (and I think it is a reasonable approach) that there is no need to perform complicated procedure with sleeping pill. It is enough that one can imagine to perform such a procedure to justify the betting assignment of the experimentalist who believe in the MWI. So, identical experiences lead to identical behavior, although the argumentation is different, which is not surprising in view of different world views.

\section{Deriving the Born Rule}

The betting assignments are according to the Born Rule or to the Born-Vaidman Rule. The rules are postulates which are added to the formalism of quantum mechanics of evolution of the wave function. In my view, addition of a postulate is well justified by its confirmation by our observations. However, I do not want to leave unnoticed a large and increasing effort to derive the Born Rule. Deutsch (1999) started a program (extended by Wallace (2012)) to derive the proper betting behavior in the framework of the MWI based on some postulates of Decision theory. The program encountered criticism of apparently circular definitions. The Deutsch- 
Wallace proof is complicated and it is not simple to understand what exactly is proved and what is assumed. I also suggested a proof, Vaidman (2012). My work was triggered by Deutsch (1999) paper, but the proof is apparently different. It is based on symmetry and relativistic causality. Apart from the fact that in the framework of collapse theory we have to postulate relativistic causality, while in the framework of the MWI it is part of the physical theory, my proof is applicable to both cases McQueen and Vaidman (2018). Very recently Sebens and Caroll (2018) proposed yet another proof. They say it follows my approach of self-locating uncertainty and relies on the assumption that action on environment cannot change outcomes of local measurements. This assumption is not very different from my relativistic causality, however, it seems to me that their proof does not hold. It is based on permutation symmetry and relies on a metaphysical approach to personal identity the justification for which I cannot understand. Sebens and Carroll manipulate the concept of probability of an observer to be in a particular world when splitting has already happened, but the wave function of the observer is identical in both worlds. Not only the mental state is identical (sufficient for numerical identity in Tappenden sense) and not only the wave function of relative variables of the body of the observer (which might happen in my sleeping pill experiment) but the whole wave function is fully identical in both worlds. The worlds are distinguished by macroscopic differences of other objects. In this case there is no matter of fact in which of the two worlds the observer is present, since it is present in both. See details in Kent (2015) and McQueen and Vaidman (2018).

Putting aside the correctness of the proofs of the Born Rule, I argue that none of them can be considered as unconditioned on any assumption. For the Born Rule in the framework of collapse theories, we need an additional postulate about collapse. It is not part of the standard formalism. A priory it needs not follow any laws of standard physical theory. We need to postulate some assumption: in my proof it is the impossibility of superluminal signaling. In the framework of the MWI we also need an assumption. The physics part, the evolution of the wave function of the Universe has to be supplemented by some law connecting it to our experience. Here the assumption maybe considered natural and minimal: everything, including our experiences supervene on the wave function of the Universe. Then, physical laws governing the evolution of the wave function are relevant to our experience too, so we might claim that no additional assumption was made. Still, in all cases there is a tacit assumption that probability (or illusion of probability) depends on the wave function.

\section{Conclusions}

Today's physics is a quantum theory and it enjoys unprecedented success explaining all observed phenomena. There are questions that do not have good answers (yet). Quantum gravity, dark matter, dark energy... It might happen that resolving these questions will require new revolutionary ideas. However, quantum mechanics apparently will remain to be the theory explaining electromagnetic interactions, the interactions which are responsible for almost everything we see in everyday life. Unphysical features of collapse are the main reasons for doubts that this is the final theory of nature. But actually there is no evidence for collapse. Apparently, it is just the philosophical difficulty to accept the consequence of accepting that there is no collapse, the existence of multiple worlds, that prevents consensus about quantum 
theory without collapse. It took time before people were ready to accept that Earth is not the center of the Universe. We also need time to accept that we are not unique and that there are many similar copies of us. We need time to establish the connection between the wellestablished mathematical part of the theory and our experience. It is an unusual situation which we did not encounter in old physical theories. Philosophers should play an important role in this project since it requires a dramatic change in our world view. Observing the rapidly increasing number of publications related to the MWI in philosophical literature I can be optimistic. I am not sure that the large effort to find a derivation of the Born rule is justified (I doubt that the MWI has significant advantage here), but this activity leads to accepting legitimacy of the MWI by physicists, and I believe that its advantage as a physical theory will bring it to consensus.

\section{Acknowledgments}

I benefited a lot from numerous discussions with Kelvin McQueen, David Albert and participants of the workshop Identity, indistinguishability and non-locality in quantum physics, Buenos Aires, June 2017. This work has been supported in part by the Israel Science Foundation Grant No 1311/14.

\section{References}

Aharonov, Y., Bohm, D. (1959). "Significance of Electromagnetic Potentials in the Quantum Theory." Physical Review, 115: 485-491.

Albert, D. Z. (2013). "Wave function realism.” Pp. 52-57, in Ney, A., \& Albert, D. Z. (eds.), The Wave Function: Essays on the Metaphysics of Quantum Mechanics. Oxford: Oxford University Press.

Deutsch, D. (1986). “Three Experimental Implications of the Everett Interpretation.” Pp. 204-214, in R. Penrose and C.J. Isham (eds.), Quantum Concepts of Space and Time, Oxford: The Clarendon Press.

Deutsch, D. (1999). "Quantum Theory of Probability and Decisions." Proceedings of the Royal Society of London. A 455: 3129-3137.

Diósi, L. (1987). “A Universal Master Equation for the Gravitational Violation of Quantum Mechanics.” Physics Letters, A 120: 377-381.

Ghirardi, G.C., Rimini, A., and Weber, T. (1986). "Unified Dynamics for Microscopic and Macroscopic Systems.” Physical Review, D34: 470-491. 
Goldstein, S. (2001), "Bohmian Mechanics.", The Stanford Encyclopedia of Philosophy, E. N. Zalta (ed.), https://plato.stanford.edu/entries/qm-bohm/.

Greaves, H. (2004). “Understanding Deutsch's Probability in a Deterministic Multiverse.” Studies in History and Philosophy of Modern Physics, 35: 423-456.

Groisman, B., Hallakoun, N., and Vaidman, L. (2013). "The Measure of Existence of a Quantum World and the Sleeping Beauty Problem.” Analysis, 73: 695-706.

Kent, A. (2015). "Does it Make Sense to Speak of Self-Location Uncertainty in the Universal Wave-function? Remarks on Sebens and Carroll." Foundations of Physics 45: 211-217.

Laplace, P. (1820). "Essai Philosophique sur les Probabilités forming the introduction to his Théorie Analytique des Probabilités." Paris: V Courcier; repr. F.W. Truscott and F.L. Emory (trans.), A Philosophical Essay on Probabilities, New York: Dover, 1951.

Lewis, D. (1986). On the Plurality of Worlds, Oxford, New York: Basil Blackwell.

Maudlin, T. (2013). "The nature of the quantum state." Pp. 126-153, in Ney, A., \& Albert, D.Z. (eds.), The Wave Function: Essays on the Metaphysics of Quantum Mechanics. Oxford: Oxford University Press.

McQueen, K. and Vaidman, L. (2018). "In Defence of the Self-Location Uncertainty Account of Probability in the Many-Worlds Interpretation” http://philsci-archive.pitt.edu/14389/

Pearle, P. (1976). "Reduction of Statevector by a Nonlinear Schrödinger Equation.” Physical Review, D13: 857-868.

Penrose, R. (1996). “On Gravity's role in Quantum State Reduction.” General Relativity and Gravitation, 28: 581-600.

Saunders, S. (2010). "Chance in the Everett Interpretation," in Many Worlds? Saunders, S., Barrett, J., Kent, A., \& Wallace, D. (eds.) Oxford: Oxford University Press.

Sebens, C.T., Carroll. C.M. (2018). "Self-locating Uncertainty and the Origin of Probability in Everettian Quantum Mechanics.” British Journal for the Philosophy of Science, 69: 25-74.

Tappenden, P. (2011). "Evidence and Uncertainty in Everett's Multiverse." British Journal for the Philosophy of Science, 62: 99-123.

Vaidman, L. (1998). "On Schizophrenic Experiences of the Neutron or Why We Should Believe in the Many-Worlds Interpretation of Quantum Theory." International Studies in the Philosophy of Science. 12: 245-261. 
Vaidman, L. (2002). "Many-Worlds Interpretation of Quantum Mechanics", The Stanford Encyclopedia of Philosophy, E. N. Zalta (ed.), https://plato.stanford.edu/entries/qmmanyworlds/.

Vaidman, L. (2012a). "Role of Potentials in the Aharonov-Bohm Effect." Physical Review, A R86: 040101.

Vaidman, L. (2012b). "Probability in the Many-Worlds Interpretation of Quantum Mechanics." Pp. 299-311, in Probability in Physics, The Frontiers Collection, Y. Ben-Menahem and M. Hemmo (eds.), Springer-Verlag, Berlin Heidelberg.

Wallace, D. (2012). The Emergent Multiverse: Quantum Theory according to the Everett Interpretation. Oxford: Oxford University Press 